\title{
Análisis Comparativo de la Participación Escolar y Bienestar Subjetivo en Estudiantes con y sin Discapacidad en Chile
}

\section{Comparative Analysis of School Participation and Subjective Well- Being of Students With and Without Disabilities in Chile}

\author{
Universidad de Concepción \\ David Sirlopú \\ Universidad del Desarrollo
}

María Ignacia Galarce Muñoz y Claudia Paz Pérez-Salas

\begin{abstract}
La participación escolar es importante, ya que impacta en el bienestar de todos los estudiantes. En el caso de los estudiantes en situación de discapacidad, el apoyo y la inclusión de docentes y compañeros en las clases regulares se asocian con niveles más altos de bienestar subjetivo. El objetivo de esta investigación fue comparar los niveles de participación escolar y de bienestar subjetivo de una muestra por conveniencia de colegios particularessubvencionados y municipales de 5 comunas en 2 regiones de Chile que contaban con un Programa de Integración Escolar. La muestra final estuvo compuesta por 120 escolares (50 con alguna discapacidad motora, visual o auditiva y 70 de ellos sin discapacidad) entre 14 y 19 años $(M=16,25, D E=1,65$ años). Los escolares fueron evaluados con las escalas de participación escolar (John-Akinola \& Nic-Gabhainn, 2014); satisfacción vital multidimensional para estudiantes (Huebner, 2001) y afecto positivo y negativo para niños y adolescentes (Watson, Clark \& Tellegen, 1988). Se presentan estadísticas descriptivas, correlaciones y comparación de medias entre los distintos grupos según situación de discapacidad. Los resultados indican una relación directa entre la participación escolar y el bienestar subjetivo de todos los estudiantes evaluados. No se detectaron diferencias en la participación escolar de estudiantes con y sin discapacidades, pero sí en el bienestar subjetivo, específicamente menores niveles de satisfacción con el sí mismo, con la familia y con los amigos en los estudiantes con discapacidad y mayor afectividad negativa en los estudiantes con trastorno motor versus discapacidad visual y auditiva
\end{abstract}

Palabras clave: participación escolar, bienestar subjetivo, discapacidad

School participation is important, since it impacts the well-being of all students. In the case of students with disabilities, support and inclusion conveyed by teachers and classmates in regular lessons are associated with higher levels of subjective well-being. The aim of this study was to compare the levels of school participation and subjective well-being of a convenience sample of subsidized private and municipal schools located in 5 districts of 2 regions of Chile that had a School Integration Program. The final sample comprised 120 schoolchildren (50 with a motor, visual, or hearing disability and 70 with no disabilities) between 14 and 19 years old $(\mathrm{M}=16.25, \mathrm{SD}=1.65$ years). Participants were evaluated with the following scales: school participation (John-Akinola \& Nic-Gabhainn, 2014), multidimensional student life satisfaction (Huebner, 2001), and positive and negative affect for children and adolescents (Watson, Clark, \& Tellegen, 1988). Descriptive statistics, correlations, and comparisons of means between the groups (according to disability status) are presented. Results indicate a direct relationship between school participation and subjective well-being in all evaluated students. No differences in school participation were found between students with and without disabilities, but subjective well-being did differ among them. Specifically, students with disabilities showed lower levels of self-satisfaction, family satisfaction, and friend satisfaction than students without disabilities; in addition, students with motor disabilities displayed higher levels of negative affect than those with visual and hearing disabilities.

Keywords: school participation, subjective well-being, disability

La Organización Mundial de la Salud (OMS, 2011) estima que más de 1000 millones de personas tienen alguna discapacidad, lo que representaría el 15\% de la población global. Sin embargo, algunos autores consideran que la prevalencia de la discapacidad podría ser aún mayor, debido a múltiples factores que

María Ignacia Galarce Muñoz y Claudia Paz Pérez-Salas, Departamento de Psicología, Universidad de Concepción, Chile; David Sirlopú, Facultad de Psicología, Universidad del Desarrollo, Concepción, Chile.

Esta investigación contó con el financiamiento del proyecto FONDECYT 1151360 otorgado al tercer autor.

La correspondencia relativa a este artículo debe ser dirigida a Claudia Paz Pérez-Salas, Departamento de Psicología, Universidad de Concepción, Víctor Lamas 1290, Concepción, Chile. E-mail: cperezs@udec.cl 
afectan su medición, tales como la definición empleada, la calidad de los datos, los métodos de análisis usados y la rigurosidad de las fuentes (Al Ju'beh, 2017).

La discapacidad puede ser conceptualizada de maneras distintas según el modelo teórico empleado (Smith \& Bundon, 2018). En este estudio se utilizó el modelo socio-relacional (Thomas, 2007), el cual entiende la discapacidad como un fenómeno que se expresa en el espacio de las relaciones sociales, en lugar de ubicar la discapacidad dentro del individuo. Desde esta perspectiva, la discapacidad se concibe como producto de la relación entre la persona y su entorno. Por ello, Paolinelli y González (2014) proponen que no se debería hablar de personas con discapacidad sino de personas en situación de discapacidad (en adelante, PeSD). De este modo, una persona con alguna discapacidad llega a conocer qué es lo que implica su situación cuando otros individuos lo tratan de manera diferente, ya sea como objeto de admiración, burla, lástima o indiferencia (Michailakis, 2003).

La Clasificación Internacional del Funcionamiento, la Discapacidad y la Salud (CIF en español), que fue aprobada por la OMS (2001), ofrece un marco teórico integral y unificado que pone énfasis en la salud y el funcionamiento de las PeSD y no en la discapacidad (Chaná \& Alburquerque, 2006). La CIF propone, por un lado, la evaluación de las personas en su funcionamiento (asociado con las funciones corporales, las actividades y la participación) y, por otro, en la discapacidad (relacionado con las deficiencias, limitación de actividades o restricción de la participación). Adicionalmente, contempla factores contextuales, que se dividen en ambientales y personales. Es, pues, un modelo que aborda dimensiones corporales, individuales y sociales de las personas y que se encuentran interrelacionadas entre sí (Fernández-López, FernándezFidalgo, Geoffrey, Stucki \& Cieza, 2009). Este constituye una evolución desde un modelo médicorehabilitador (que asimila la deficiencia a la enfermedad) a un modelo biopsicosocial (que incluye uno de los presupuestos centrales del modelo social de la discapacidad como es la importancia que tienen los factores sociales a la hora de definir el origen de la discapacidad (Palacios, 2008).

De este modo, la Convención Internacional sobre los Derechos de las Personas con Discapacidad (Organización de las Naciones Unidas, 2006) establece que:

Las personas con discapacidad incluyen a aquellas que tengan deficiencias físicas, mentales, intelectuales o sensoriales a largo plazo que, al interactuar con diversas barreras, puedan impedir su participación plena y efectiva en la sociedad, en igualdad de condiciones con las demás. (p. 4)

En Chile, según lo reportado en el último Estudio Nacional de la Discapacidad (Chile, Ministerio de Desarrollo Social, 2016), viven un poco más de 2 millones y medio de PeSD (20\% del total de la población). De ellas, el 11,7\% presenta discapacidad leve o moderada y el resto, discapacidad severa. En cuanto a la población que comprende a personas entre los 2 y 17 años, el 5,8\% presenta alguna discapacidad, correspondiendo la mayor prevalencia a la discapacidad intelectual $(21,5 \%)$, seguida por la motora $(9,6 \%)$, la visual $(2,8 \%)$ y la auditiva $(2,2 \%)$. Finalmente, el $96 \%$ de menores de edad con discapacidad asiste a un establecimiento escolar.

\section{Inclusión y Personas en Situación de Discapacidad}

Diferentes investigaciones han reportado que existen creencias erróneas sobre las PeSD, las que se constituyen en barreras para su inclusión en la sociedad (Bunch \& Valeo, 2004; Hein, Grumm \& Fingerle, 2011; Rodríguez Martín \& Álvarez Arregui, 2013). Estas creencias promueven la formación de prejuicios y estereotipos hacia esta minoría, lo que tiene un efecto negativo en el bienestar y calidad de vida de sus miembros (Polo Sánchez, Fernández Jiménez \& Díaz Batanero, 2011) y obstaculiza su plena participación en las distintas esferas de la vida y el logro de sus objetivos (Antonak \& Livneh, 2000).

En el ámbito de la educación, durante varias décadas las PeSD fueron tratadas como un grupo con déficits o patologías que debían ser curadas o corregidas. Actualmente, esta situación ha cambiado drásticamente en muchos países, donde la diversidad se valora y defiende, debido al reconocimiento de su importancia para el desarrollo de comunidades escolares seguras y respetuosas (Tamayo Rozas, Carvallo Arrau, Sánchez Cornejo, Besoaín-Saldaña \& Rebolledo Sanhueza, 2018). En Chile, desde 1990 se llevó a cabo una serie de reformas educativas cuyo propósito fue mejorar la equidad y la calidad del sistema educacional, mediante la atención a las diferencias y la discriminación a favor de los¹/as más vulnerables

${ }^{1}$ Los autores asumen la importancia de la distinción lingüística de género. Sin embargo, para facilitar la lectura del texto, en adelante, esta será obviada. 
(Ramos, 2013; Tenorio, 2011). Una consecuencia positiva de esta corriente fue la aparición del Programa de Integración Escolar (PIE). A través del PIE, los establecimientos educativos obtienen los recursos humanos y materiales para dar respuesta a las necesidades educativas especiales de niños, niñas y adolescentes en situación de discapacidad o con otros trastornos en la educación regular (Godoy, Meza \& Salazar, 2004).

Si bien se ha observado una evolución en la normativa respecto a la forma en que se entregan los apoyos estatales, estos todavía siguen dependiendo de diagnósticos individuales de los estudiantes y dejan de lado la transformación de la cultura escolar (Muñoz Villa, López Cruz \& Assaél, 2015). Ante esto, surge el desafío de perfeccionar la normativa actual, avanzando, además, en la coherencia entre el paradigma inclusivo y los procesos de formación para alcanzar las transformaciones necesarias para garantizar de manera efectiva el derecho a la educación de todos (Manghi et al., 2012). Por otra parte, se hace necesario sensibilizar a los futuros docentes durante la etapa de formación, sobre todo, en cómo sus creencias sobre el alumnado influyen en el rendimiento y comportamiento de sus estudiantes (Sánchez Bravo, Díaz Flores, Sanhueza Henríquez \& Friz Carrillo, 2008).

\section{Participación Escolar y Discapacidad}

La participación en actividades escolares se considera una parte vital del desarrollo de los alumnos y alumnas, especialmente el de aquellos que se encuentran en situación de discapacidad. La participación de las PeSD en su colegio les permite desarrollar habilidades y competencias, formar amistades y relaciones, expresar creatividad, desarrollar una identidad propia y determinar el significado y propósito en la vida, todo lo cual, tiene un impacto positivo en su bienestar (King et al., 2003; Law, 2002).

La literatura reporta diferencias de participación según los tipos de discapacidad. Así, los estudiantes con discapacidad motora presentan menores niveles de participación en el ámbito educativo. Esto parece responder a varios factores, tales como las barreras físicas que coartan su desplazamiento y sus niveles de autonomía (Eriksson, Welander \& Granlund, 2007; Morris, Kurinczuk, Fitzpatrick \& Rosenbaum, 2006), la falta de conocimiento de las escuelas sobre cómo adaptar el plan de estudios y los métodos de instrucción (Hemmingsson \& Borell, 2000, 2002), la escasez del equipo apropiado y la cooperación limitada entre personal de la escuela y servicios de apoyo (Egilson \& Coster, 2004; Hemmingsson, Gustavsson \& Townsend, 2007) y, en el caso específico de la parálisis cerebral, una alta comorbilidad con otras limitaciones cognitivas, perceptuales y/o comunicacionales que pueden dificultar la interacción con otras personas (Bax et al., 2005).

La participación escolar, en el caso de los alumnos que presentan discapacidad auditiva, se ve mermada por distintos factores. Por ejemplo, este grupo presenta un deficiente manejo de la lengua oral empleada por su entorno (Ibáñez, Becerra, López, Sirlopú \& Cornejo, 2005). Esta limitación, según Domínguez (2009), genera dos desafíos en el ámbito educacional, no siempre adecuadamente resueltos. El primero es que los docentes conozcan y dominen la lengua de señas utilizada por sus estudiantes y la usen eficientemente en los procesos de enseñanza-aprendizaje. El segundo es que los estudiantes oyentes y no oyentes puedan interactuar adecuadamente. De este modo, resulta relevante que los compañeros oyentes y los docentes sean sensibles a las necesidades comunicativas de los estudiantes sordos y sean capaces de modificar sus estrategias comunicativas para facilitar así sus interacciones (Domínguez, 2009).

En lo que respecta a la participación del alumno con discapacidad visual, esta se ve interferida principalmente por su dificultad para acceder a la información a través del sentido de la vista (Torres Gutiérrez, 2006).

\section{Bienestar Subjetivo y Discapacidad}

El bienestar subjetivo se entiende como una evaluación global de la propia vida, formada por distintos componentes (satisfacción vital, afecto positivo y ausencia de afecto negativo), a la que se le agrega la satisfacción con diferentes dominios en los que la persona participa (Diener et al., 2009). La literatura reporta que las experiencias escolares positivas y la eficacia escolar contribuyen como factores protectores para el bienestar de niños y adolescentes (Condly, 2006). Lo anterior ocurre sobre todo en escuelas con clima positivo (Konu, Lintonen \& Rimpelä, 2002), que promueven un sentido de pertenencia (Matos \& Carvalhosa, 2001) y poseen un liderazgo positivo y personal motivado (Pianta, 2006). 
Cuando un estudiante en situación de discapacidad es capaz de desarrollar habilidades durante el proceso escolar y consigue objetivos en el tiempo, este tiende a percibir mayor grado de satisfacción con su vida (Steptoe, Deaton \& Stone, 2015). Asimismo, el apoyo y la inclusión de los docentes y los compañeros hacia las PeSD en las clases regulares se asocian con niveles más altos de bienestar subjetivo (Opdenakker \& Van Damme, 2000).

$\mathrm{Al}$ igual que en otras áreas del conocimiento, las investigaciones sobre escolares en situación de discapacidad carecen de un enfoque de género (Tinklin, Croxford, Ducklin \& Frame, 2003). De este modo, cuando los colegios cuentan con un currículo que incorpora esta temática, este no suele incluir la gran variedad de obstáculos diferenciales a los que se enfrentan niños y niñas en situación de discapacidad (ChildFund International, 2016). Las investigaciones sobre este tema han mostrado que, independiente del género, las PeSD están expuestas a mayor discriminación y menores oportunidades de participación, pero suelen ser las mujeres las que son doblemente discriminadas: por su género y su situación (OMS, 2011).

En cuanto a los tipos de discapacidad, varias investigaciones (Carona, Moreira, Silva, Crespo \& Canavarro, 2014; Nunes, Pretzlik \& Olsson, 2001) reportan que los jóvenes con discapacidad motora y discapacidad auditiva presentan niveles más bajos en la dimensión de apoyo social percibido, en comparación con sus pares sin discapacidad. Las personas con discapacidad visual, por su parte, muestran una mayor satisfacción con el apoyo social, proporcionado principalmente por la familia, en comparación con sus pares sin discapacidad (Kef, Hox \& Habekothé, 2000). Respecto de la satisfacción vital de las personas con deficiencias sensoriales, se han reportado mayores niveles de satisfacción vital, en comparación al de las personas con discapacidad física (Mehnert, Krauss, Nadler \& Boyd, 1990).

\section{El Problema}

Un alto grado de participación en actividades escolares es esencial para el aprendizaje y la satisfacción con la vida (Simeonsson, Carlson, Huntington, McMillen \& Brent, 2001). No obstante, se ha encontrado que los escolares en situación de discapacidad presentan más barreras para participar, debido a que poseen condiciones que hacen que la satisfacción de las necesidades psicológicas básicas sea más cambiante, ya que dependen más de los demás y del apoyo del contexto para satisfacer esas necesidades que las personas sin discapacidad, lo cual afecta su bienestar subjetivo (Mancini, Coster, Trombly \& Heeren, 2000). Aunque es posible encontrar investigaciones sobre el bienestar subjetivo en PeSD, una mínima parte de estos se refiere a la importancia de la participación en el ámbito educativo y cómo esta impacta en su bienestar subjetivo y calidad de vida en general (Gómez-Vela \& Verdugo, 2004). Así, se hace necesario profundizar en esta temática, toda vez que la participación en el proceso educativo se considera como un pilar fundamental en el bienestar de los estudiantes en situación de discapacidad (Suriá Martínez, 2016).

En consecuencia, es importante conocer la experiencia de estos estudiantes respecto a su participación en la escuela y su relación con el bienestar subjetivo para que los establecimientos educacionales puedan proponer intervenciones específicas que satisfagan las necesidades educativas especiales de sus estudiantes en situación de discapacidad y, por lo tanto, promuevan su funcionamiento positivo (Moreira et al., 2015).

Considerando lo anteriormente expuesto, en este estudio se plantearon cinco hipótesis. Primero, existe una relación directa entre la participación escolar y el bienestar subjetivo en las distintas esferas de la vida de estudiantes en situación de discapacidad (H1). Segundo, los estudiantes hombres en situación de discapacidad presentan mayores niveles de participación escolar que las estudiantes mujeres (H2). Tercero, los estudiantes en situación de discapacidad de mayor nivel socioeconómico (NSE) presentan mayores niveles de participación escolar que el resto de sus pares (H3). Cuarto, los estudiantes en situación de discapacidad presentan menores niveles de participación escolar que los estudiantes sin discapacidad, siendo los escolares con discapacidad motora los que menos participan (H4). Quinto, los estudiantes sin discapacidad presentan mayores niveles de bienestar subjetivo, en comparación a los estudiantes en situación de discapacidad. En este grupo, los que presentan discapacidad motora evidencian menores puntajes en bienestar subjetivo (H5).

\section{Método}

Se utilizó un diseño no experimental, transversal-correlacional. 


\section{Participantes}

Participó un total de 120 personas, con las cuales se conformaron cuatro grupos de estudiantes: (1) sin discapacidad ( $n=70)$; (2) con discapacidad motora $(n=18)$; (3) con discapacidad visual $(n=17)$; y (4) con discapacidad auditiva $(n=15)$. Todos los estudiantes fueron contactados en seis colegios particulares subvencionados y 16 municipales de cinco comunas de dos regiones de Chile que contaban con PIE: Chillán, Ñiquén, San Carlos, Pinto y Bulnes (Región del Ñuble) y Los Ángeles (Región del Biobío).

El tipo de muestreo para los colegios fue de tipo no probabilístico por conveniencia y para los grupos en situación de discapacidad fue de tipo intencionado, según el cumplimiento de los criterios de inclusión o exclusión (Hernández Sampieri, Fernández-Collado \& Baptista Lucio, 2006). El grupo de comparación (submuestra sin discapacidad) fue escogido con la estrategia de emparejamiento, teniendo en cuenta curso, edad y sexo de cada estudiante de los grupos en situación de discapacidad (Hernández Sampieri et al., 2006). No obstante, se incluyó a un número mayor de estudiantes sin necesidades educativas especiales para así obtener una muestra mayor y con ello aumentar la potencia del estudio (Hair, Anderson, Tatham \& Black, 2005).

Los criterios de inclusión de la muestra de estudiantes en situación de discapacidad fueron: (a) ser estudiante de los colegios contactados, (b) tener entre 14 a 19 años de edad, (c) estar cursando la enseñanza media, (d) pertenecer al PIE de su establecimiento, (e) tener diagnóstico de discapacidad motora (submuestra con discapacidad motora), discapacidad auditiva (submuestra con discapacidad auditiva) o discapacidad visual (submuestra con discapacidad visual). Por su parte, los criterios de inclusión del grupo de estudiantes sin discapacidad fueron: (a) ser estudiante de los colegios contactados, (b) tener entre 14 y 19 años, (c) estar cursando la enseñanza media y (d) no poseer ninguna discapacidad ni pertenecer al PIE de su establecimiento educacional. Como criterio de exclusión para todas las submuestras se consideró la presencia de trastornos psiquiátricos (esquizofrenia, trastorno bipolar, trastorno del espectro autista), multidéficit o discapacidad intelectual.

En la Tabla 1 se puede observar la muestra final, considerando el promedio de edad, el sexo y el NSE, de los alumnos sin ninguna discapacidad (grupo de comparación), con discapacidad motora, con discapacidad auditiva y con discapacidad visual.

Tabla 1

Características de los Participantes según Condición

\begin{tabular}{|c|c|c|c|c|c|c|c|c|c|}
\hline Condición & $N$ & $\begin{array}{l}\text { Hombres } \\
(n=66)\end{array}$ & $\begin{array}{l}\text { Mujeres } \\
(n=54)\end{array}$ & $\begin{array}{c}\text { Edad } \\
M \\
(D E)\end{array}$ & $\begin{array}{l}\text { Rango } \\
\text { edad }\end{array}$ & $\begin{array}{l}\mathrm{NSE} \\
\text { bajo }\end{array}$ & $\begin{array}{l}\text { NSE } \\
\text { medio } \\
\text { bajo }\end{array}$ & $\begin{array}{l}\mathrm{NSE} \\
\text { medio } \\
\text { alto }\end{array}$ & $\begin{array}{l}\text { NSE } \\
\text { alto }\end{array}$ \\
\hline $\begin{array}{l}\text { Escolares sin } \\
\text { discapacidad }\end{array}$ & 70 & 38 & 32 & $\begin{array}{l}16,61 \\
(0,18)\end{array}$ & $14-19$ & $6(9 \%)$ & $16(23 \%)$ & $31(44 \%)$ & $17(24 \%)$ \\
\hline $\begin{array}{l}\text { Escolares con } \\
\text { discapacidad motora }\end{array}$ & 18 & 8 & 10 & $\begin{array}{l}15,72 \\
(0,43)\end{array}$ & $14-19$ & $4(22 \%)$ & $3(17 \%)$ & $7(39 \%)$ & $4(22 \%)$ \\
\hline $\begin{array}{l}\text { Escolares con } \\
\text { discapacidad } \\
\text { auditiva }\end{array}$ & 17 & 13 & 4 & $\begin{array}{l}15,53 \\
(0,39)\end{array}$ & $14-19$ & $0(0 \%)$ & $3(18 \%)$ & $12(70 \%)$ & $2(12 \%)$ \\
\hline $\begin{array}{l}\text { Escolares con } \\
\text { discapacidad visual }\end{array}$ & 15 & 7 & 8 & $\begin{array}{l}16,07 \\
(0,48)\end{array}$ & $14-19$ & $0(0 \%)$ & $7(47 \%)$ & $6(40 \%)$ & $2(13 \%)$ \\
\hline
\end{tabular}

$\mathrm{Al}$ analizar la composición de la muestra, no se detectaron diferencias en la proporción de hombres y mujeres según grupo, $\chi^{2}(3, N=120)=4,41, p=0,220$, en el promedio de edad según el grupo $(F(3,112)=2,59$, $p=0,056)$ y el sexo $(F(1,112)=0,0004, p=0,983)$ de los participantes. Tampoco se detectaron diferencias en la proporción de estudiantes en cada NSE por grupo, $\left.\chi^{2}(9, N=120)=14,78, p=0,097\right)$. 


\section{Instrumentos}

Escala de Participación Escolar (School Participation Scale). Elaborada por John-Akinola y NicGabhainn (2014) y consta de 25 ítems que miden cuatro dimensiones de la participación escolar: (a) participación en las decisiones y reglamento de la escuela (6 ítems: "Los alumnos participan en la elaboración del reglamento del colegio"), (b) participación en actividades extra-académicas tales como educación física, artes, música (7 ítems: "Me entretengo realizando estas actividades"), (c) participación en eventos escolares tales como el día del deporte (6 ítems: "Los alumnos participan de la planificación de eventos escolares") y (d) percepción positiva de la participación escolar (6 ítems: "En mi colegio, todos los alumnos tienen la posibilidad de participar"). Cada ítem se responde en una escala Likert de 5 puntos $(1=$ nunca; $5=$ siempre), mostrando un mayor puntaje una mayor participación.

Este instrumento fue validado en escolares chilenos por Pérez-Salas, Sirlopú, Cobo y Awad (2019). El coeficiente Omega obtenido por dichos autores para la primera dimensión fue 0,85, para la segunda, 0,83, para la tercera, 0,86 y para la cuarta, 0,87. En lo que respecta a la validez de constructo, el análisis factorial confirmatorio (AFC) confirmó la presencia de un factor de participación escolar general, además de las cuatro dimensiones contempladas en la escala, $\chi^{2}(244, N=1428)=1412,28, \mathrm{p}<0,001$, CFI $=0,97$, RMSEA $=0,05$ ), coherente con lo propuesto por los creadores del instrumento (John-Akinola \& NicGabhainn, 2014).

Escala Multidimensional de Satisfacción Vital para Estudiantes (Multidimensional Students' Life Satisfaction Scale, SLSS). Fue desarrollada por Huebner (2001) y consta de 40 ítems que miden cinco dimensiones de la satisfacción vital: familia (7 ítems: "Disfruto estar en casa con mi familia"), amigos (9 ítems: "Mis amigos me tratan bien"), escuela (8 ítems: "La escuela es interesante"), entorno (9 ítems: "Me gustan mis vecinos") y sí mismo (7 ítems: "Soy una persona entretenida"). Cada ítem se responde con una escala Likert de 5 puntos $(1=$ muy en desacuerdo; $5=$ muy de acuerdo), por lo que puntajes altos indican mayor nivel de satisfacción. Respecto de su confiabilidad, Huebner (2001) informa coeficientes alfa de Cronbach entre 0,70 y 0,90 para las distintas dimensiones. En Chile, este instrumento fue validado en población infantil por Chavarría y Barra (2014), quienes reportaron coeficientes alfa de Cronbach entre 0,77 y 0,87 para las distintas dimensiones.

Escala de Afecto Positivo y Negativo para Niños y Adolescentes (PANASN). Se utilizó una versión en español del Positive and Negative Affect Schedule (PANAS) de Watson, Clark y Tellegen (1988) para adolescentes adaptado por Sandín (2003). Esta escala consta de 20 ítems (10 miden afecto positivo: "Me intereso por la gente o por las cosas" y 10 afecto negativo: "Me siento culpable"). Cada ítem se responde con una escala Likert de 3 puntos $(1=$ nunca; $3=$ muchas veces $)$. La versión española del PANAS obtuvo coeficientes alfa de Cronbach superiores a 0,80 para ambas dimensiones (Sandín et al., 1999). En Chile, González, Oyanadel y Peñate (2018) validaron esta escala en escolares chilenos y obtuvieron índices de consistencia interna alfa de Cronbach de 0,84 para la subescala afecto positivo y 0,85 para la subescala afecto negativo. A través de AFC se corroboró una configuración bidimensional, $\chi^{2}(160, N=467)=328,72, p<0,05$, $\mathrm{CFI}=0,95$, RMSEA $=0,05)$.

\section{Procedimiento}

Esta investigación contó con la autorización del comité de ética del Departamento de Psicología de la Universidad de Concepción.

Una vez obtenida la autorización institucional de las direcciones municipales de educación y establecimientos educacionales contactados, se invitó a participar a los estudiantes que cumplían con los criterios de inclusión. Se envió el consentimiento informado al adulto responsable de los escolares, para que autorizara la aplicación de los instrumentos. Cuando fueron autorizados, se solicitó el asentimiento de cada participante para colaborar con la investigación. Una profesora diferencial colaboró en la explicación del asentimiento a los estudiantes en situación de discapacidad, en caso de requerirlo.

Las evaluaciones fueron realizadas por la primera investigadora y se efectuaron de manera grupal para los estudiantes sin discapacidad en una sala y horario dispuestos por cada colegio. El tiempo de aplicación promedio fue aproximadamente 45 minutos. Con respecto a las evaluaciones de los estudiantes en situación de discapacidad, estas fueron realizadas de manera individual en una sala asignada por el establecimiento para ese fin. Además, se contó con el apoyo de una profesora diferencial, quien registró las respuestas de 
algunos estudiantes. Así, en el caso de las personas con discapacidad auditiva que no podían leer de manera fluida, la profesora especialista tradujo las preguntas a lengua de señas chilena (LSCh) y los alumnos señalaban su opción de respuesta. Para los estudiantes con discapacidad motora que no podían escribir, se les leyeron los instrumentos y luego la profesora especialista registró sus respuestas. En lo que se refiere a estudiantes con discapacidad visual, se presentó el instrumento de manera oral y luego la profesora especialista registró sus respuestas.

\section{Análisis de Datos}

Previo al análisis de los datos, se testeó el cumplimiento de los supuestos de las técnicas paramétricas: distribución normal con asimetría y curtosis y prueba de Shapiro Wilks y homogeneidad de varianzas con la Prueba de Levene.

Una vez evaluado el cumplimiento de los supuestos para efectuar análisis paramétricos, se realizaron correlaciones bivariadas utilizando el coeficiente de correlación lineal de Pearson para analizar la asociación entre la participación escolar y el bienestar subjetivo de los estudiantes. Para comparar los niveles de participación escolar según sexo, NSE y tipo de discapacidad se realizaron análisis de varianza (ANOVA) para cada una de las variables contempladas, utilizando el ajuste de Welch para las dimensiones que presentaron heterocedasticidad. Finalmente, para comparar el bienestar subjetivo según tipo de discapacidad, se realizaron tres contrastes planificados, a través de la prueba $t$ de Student. El primero comparó al grupo sin discapacidad versus todos los grupos con discapacidad juntos; el segundo contraste comparó al grupo con discapacidad motora versus con discapacidad visual y auditiva juntos y, finalmente, el último contraste comparó el bienestar subjetivo del grupo con discapacidad visual versus el grupo con discapacidad auditiva. La lógica de realizar comparaciones planificadas, en vez de comparaciones post-hoc tradicionales, obedece a las hipótesis previas que tenía esta investigación, en la que resulta relevante comparar el grupo sin discapacidad versus todos los grupos con discapacidad juntos y luego evaluar si el grupo con discapacidad motora era el que menos bienestar subjetivo tenía, en comparación a los otros dos grupos en situación de discapacidad. De acuerdo con Field (2009), este tipo de comparación es la indicada cuando se tienen hipótesis direccionales claras al inicio de la investigación, dado que poseen mayor potencia estadística para detectar el efecto deseado.

Todos los análisis fueron realizados con el software SPSS, versión 25 (IBM, 2017).

\section{Resultados}

Los puntajes de participación y bienestar subjetivo en todas las dimensiones analizadas se distribuyeron de manera normal entre los grupos (asimetría y curtosis $<|2|$ y valores Shapiro Wilks con $p>0,05$ ). Asimismo, la mayoría de las dimensiones en ambas variables cumplieron el supuesto de homogeneidad de las varianzas, a excepción de las dimensiones Participación en Eventos Escolares, Levene(3, 116) = 0,30, $p=0,004$, Satisfacción Vital con la Familia, Levene $(3,116)=4,15, p=0,008$, Satisfacción Vital con los Amigos, Levene $(3,116)=4,51$, $p=0,005$, y Satisfacción Vital con el Sí Mismo, Levene $(3,116)=2,99, p=0,034$.

Los promedios y desviaciones estándar de la participación escolar y bienestar subjetivo de los grupos sin y con discapacidades se presentan en la Tabla 2, según condición y dimensión evaluada.

En la Tabla 3 se aprecian las correlaciones entre la participación escolar total y la percepción positiva que tienen los estudiantes de las posibilidades de participar en su escuela. Los resultados muestran una asociación moderada y directa (tanto en el grupo sin discapacidad como en los grupos en situación de discapacidad) con el afecto positivo global, la satisfacción de sí mismo y su satisfacción con la familia. En el caso de los estudiantes en situación de discapacidad, estas variables están asociadas, además y de manera importante, con su satisfacción en la escuela. La participación en actividades escolares en ambos grupos se asocia a una mayor satisfacción con el sí mismo, familia y afecto positivo. En el caso de los estudiantes en situación de discapacidad, también se asocia con una mayor satisfacción con la escuela. 
Tabla 2

Participación Escolar y Bienestar Subjetivo según Condición

\begin{tabular}{|c|c|c|c|c|c|c|c|c|c|c|}
\hline \multirow[t]{2}{*}{$\begin{array}{l}\text { Participación escolar y bienestar } \\
\text { subjetivo }\end{array}$} & \multicolumn{2}{|c|}{$\begin{array}{l}\text { Escolares sin } \\
\text { discapacidad }\end{array}$} & \multicolumn{2}{|c|}{$\begin{array}{l}\text { Escolares con } \\
\text { discapacidad * }\end{array}$} & \multicolumn{2}{|c|}{$\begin{array}{c}\text { Escolares con } \\
\text { discapacidad } \\
\text { motora }\end{array}$} & \multicolumn{2}{|c|}{$\begin{array}{c}\text { Escolares con } \\
\text { discapacidad } \\
\text { auditiva }\end{array}$} & \multicolumn{2}{|c|}{$\begin{array}{c}\text { Escolares con } \\
\text { discapacidad } \\
\text { visual }\end{array}$} \\
\hline & $M$ & $D E$ & $M$ & $D E$ & $M$ & $D E$ & $M$ & $D E$ & $M$ & $D E$ \\
\hline $\begin{array}{l}\text { Participación en decisiones y normas } \\
\text { escolares }\end{array}$ & 2,74 & 0,61 & 2,59 & 0,61 & 2,40 & 0,74 & 2,61 & 0,49 & 2,79 & 0,52 \\
\hline $\begin{array}{l}\text { Participación en actividades escolares } \\
\text { (extra-académicas) }\end{array}$ & 2,82 & 0,60 & 2,75 & 0,69 & 2,61 & 0,65 & 2,76 & 0,72 & 2,90 & 0,71 \\
\hline Participación en eventos escolares & 2,99 & 0,57 & 2,78 & 0,76 & 2,47 & 0,93 & 3,13 & 0,54 & 2,77 & 0,61 \\
\hline $\begin{array}{l}\text { Percepción positiva de las } \\
\text { posibilidades de participación escolar }\end{array}$ & 3,03 & 0,70 & 2,81 & 0,75 & 2,84 & 0,85 & 2,78 & 0,75 & 2,80 & 0,66 \\
\hline Participación escolar total & 2,89 & 0,50 & 2,73 & 0,57 & 2,58 & 0,72 & 2,82 & 0,48 & 2,82 & 0,47 \\
\hline Satisfacción vital con la familia & 4,24 & 0,58 & 3,90 & 0,70 & 3,63 & 0,92 & 3,99 & 0,50 & 4,12 & 0,51 \\
\hline Satisfacción vital con los amigos & 4,13 & 0,51 & 3,73 & 0,77 & 3,39 & 0,85 & 3,90 & 0,56 & 3,94 & 0,79 \\
\hline Satisfacción vital con la escuela & 3,46 & 1,59 & 3,67 & 0,73 & 3,43 & 0,67 & 3,89 & 0,86 & 3,72 & 0,57 \\
\hline Satisfacción vital con el entorno & 3,65 & 0,67 & 3,67 & 0,80 & 3,55 & 0,73 & 3,91 & 0,83 & 3,54 & 0,83 \\
\hline Satisfacción vital con el sí mismo & 4,08 & 0,68 & 3,83 & 0,65 & 3,79 & 0,85 & 3,83 & 0,61 & 3,87 & 0,41 \\
\hline Afecto positivo & 2,36 & 0,29 & 2,25 & 0,39 & 2,25 & 0,45 & 2,16 & 0,36 & 2,33 & 0,35 \\
\hline Afecto negativo & 1,89 & 0,36 & 1,96 & 0,40 & 2,11 & 0,42 & 1,92 & 0,40 & 1,83 & 0,37 \\
\hline
\end{tabular}

* Todos los grupos con discapacidad juntos.

Tabla 3

Correlaciones entre Participación Escolar y Bienestar Subjetivo en Escolares con y sin Discapacidad

\begin{tabular}{|c|c|c|c|c|c|c|c|c|}
\hline & & & & Bienest & r subjetiv & $\mathrm{y}$ afecto & & \\
\hline Participación & escolar & Familia & Amigos & Facuela & Fntorno & Sí & Afecto & Afecto \\
\hline $\begin{array}{l}\text { Participación en } \\
\text { decisiones y normas }\end{array}$ & Sin discapacidad & 0,20 & 0,01 & 0,08 & 0,18 & $0,34^{* *}$ & $0,26^{*}$ & $-0,01$ \\
\hline escolares & Con discapacidad & 0,14 & 0,16 & $0,45^{* *}$ & 0,12 & $-0,01$ & 0,26 & $-0,28^{*}$ \\
\hline Participación en & Sin discapacidad & $0,26^{*}$ & 0,20 & 0,16 & 0,22 & $0,42^{* *}$ & $0,35^{* *}$ & $-0,27^{*}$ \\
\hline (extra-académicas) & Con discapacidad & $0,28^{*}$ & 0,17 & $0,46^{* *}$ & 0,12 & $0,34^{*}$ & $0,38^{* *}$ & $-0,05$ \\
\hline Participación en eventos & Sin discapacidad & 0,13 & 0,22 & 0,13 & $0,34^{* *}$ & $0,39^{* *}$ & $0,41^{* *}$ & $-0,14$ \\
\hline escolares & Con discapacidad & $0,31^{*}$ & $0,29^{*}$ & $0,29^{*}$ & 0,19 & 0,17 & $0,34^{*}$ & $-0,07$ \\
\hline $\begin{array}{l}\text { Percepción positiva de } \\
\text { las posibilidades de }\end{array}$ & Sin discapacidad & $0,26^{*}$ & $0,33^{* *}$ & 0,14 & $0,34^{* *}$ & $0,45^{\text {** }}$ & $0,29^{*}$ & $-0,19$ \\
\hline participación escolar & Con discapacidad & $0,28^{*}$ & 0,25 & $0,54^{* *}$ & $-0,01$ & $0,42^{* *}$ & $0,45^{* *}$ & $-0,15$ \\
\hline Total escala & Sin discapacidad & $0,27^{*}$ & $0,24^{*}$ & 0,16 & $0,34^{* *}$ & $0,51^{* *}$ & $0,41^{* *}$ & $-0,20$ \\
\hline & Con discapacidad & $0,33^{*}$ & 0,27 & $0,53^{* *}$ & 0,13 & $0,29^{*}$ & $0,45^{* *}$ & $-0,16$ \\
\hline
\end{tabular}

${ }^{*} p<0,05 ;{ }^{* *} p<0,001$

En la Tabla 3 se observa que la participación en eventos escolares (en el caso de los estudiantes en situación de discapacidad), se asocia con la satisfacción vital con los amigos, a diferencia del grupo sin 
discapacidad. En este grupo, la percepción positiva de las oportunidades de participación y la participación total están asociadas a la satisfacción con amigos. Asimismo, la participación en eventos escolares también se relaciona con la satisfacción con la familia, la escuela y con el afecto positivo en el grupo de estudiantes con discapacidad y, en el grupo sin discapacidad, solo se asocia con la satisfacción con el entorno, el sí mismo y el afecto positivo. Finalmente, la participación en decisiones y normas escolares se correlaciona directa y moderadamente con la satisfacción vital en la escuela e inversamente con el afecto negativo para el grupo con discapacidad y con una mayor satisfacción vital con el sí mismo y mayor afecto positivo para los estudiantes sin discapacidades.

Al comparar los niveles de participación escolar de las PeSD según sexo y NSE, se encontraron diferencias entre hombres y mujeres en la dimensión participación en decisiones y normas escolares, $F(1,42)=11,28, p=0,002 \eta_{\mathrm{p}}^{2}=0,21,95 \%$ IC $\left.0,110,0,770\right]$, participación en eventos escolares, $F(1,42)=4,21$, $p=0,046, \eta_{\mathrm{p}}{ }^{2}=0,091,95 \%$ IC [-0,044, 0,804], y en la participación escolar total, $F(1,42)=5,18, p=0,028$, $\eta_{\mathrm{p}}{ }^{2}=0,11,95 \%$ IC [-0,032, 0,612]. Específicamente, los estudiantes hombres en situación de discapacidad presentan mayores niveles de participación escolar en decisiones y normas escolares $(M=2,78, D E=0,49)$, en comparación con las estudiantes mujeres con la misma condición $(M=2,34, D E=0,67)(d=0,76)$; mayor participación en eventos escolares $(M=2,95, D E=0,63)$ que las mujeres $(M=2,57, D E=0,86)(d=0,51)$ y mayor participación escolar total $(M=2,86, D E=0,46)$ que las mujeres en esa condición $(M=2,57, D E=0,67)$ $(d=0,51)$. Al comparar los niveles de participación escolar de las PeSD según NSE, no se encontraron diferencias en ninguna de las dimensiones evaluadas $\left(\mathrm{F}(12,123)=0,782, p=0,668, \eta_{\mathrm{p}}{ }^{2}=0,071\right)$, ni se detectaron efectos de interacción entre sexo y $\operatorname{NSE}\left(\mathrm{F}(12,123)=0,996, p=0,457, \eta_{\mathrm{p}}{ }^{2}=0,089\right)$. Tampoco hubo diferencias en ninguna de las dimensiones de la participación escolar según tipo de discapacidad.

Al comparar todas las dimensiones del bienestar subjetivo en los cuatro grupos considerados en la muestra (sin discapacidad, con discapacidad visual, con discapacidad auditiva y con discapacidad motora), a través de la prueba ANOVA, solo se encontraron diferencias en el ámbito de la familia y amigos (ver Tabla 4).

Tabla 4

Comparación de Medias del Bienestar Subjetivo según Condición

\begin{tabular}{|c|c|c|c|c|c|c|}
\hline & & Suma de cuadrados & gl & Media cuadrática & $F$ & $p$ \\
\hline \multirow{3}{*}{$\begin{array}{l}\text { Satisfacción vital- } \\
\text { Familia }\end{array}$} & Entre grupos & 5,70 & 3 & 1,90 & \multirow[t]{3}{*}{3,02} & \multirow[t]{3}{*}{0,003} \\
\hline & Dentro de grupos & 44,84 & 34,29 & 1,31 & & \\
\hline & Total & 50,55 & 37,29 & & & \\
\hline \multirow{3}{*}{$\begin{array}{l}\text { Satisfacción vital- } \\
\text { Amigos }\end{array}$} & Entre grupos & 7,85 & 3 & 2,62 & \multirow[t]{3}{*}{4,40} & \multirow[t]{3}{*}{$<0,001$} \\
\hline & Dentro de grupos & 43,92 & 31,13 & 1,41 & & \\
\hline & Total & 51,77 & 34,13 & & & \\
\hline \multirow{3}{*}{$\begin{array}{l}\text { Satisfacción vital- } \\
\text { Entorno }\end{array}$} & Entre grupos & 1,49 & 3 & 0,49 & \multirow[t]{3}{*}{0,94} & \multirow[t]{3}{*}{0,424} \\
\hline & Dentro de grupos & 60,78 & 116 & 0,52 & & \\
\hline & Total & 62,27 & 119 & & & \\
\hline \multirow{3}{*}{$\begin{array}{l}\text { Satisfacción vital- } \\
\text { Escuela }\end{array}$} & Entre grupos & 3,22 & 3 & 0,64 & \multirow[t]{3}{*}{1,67} & \multirow[t]{3}{*}{0,600} \\
\hline & Dentro de grupos & 198,67 & 116 & 0,38 & & \\
\hline & Total & 201,88 & 119 & & & \\
\hline \multirow{3}{*}{$\begin{array}{l}\text { Satisfacción vital- } \\
\text { Sí mismo }\end{array}$} & Entre grupos & 1,92 & 3 & 0,61 & \multirow{3}{*}{1,36} & \multirow{3}{*}{0,245} \\
\hline & Dentro de grupos & 52,30 & 36,91 & 1,42 & & \\
\hline & Total & 54,22 & 39,91 & & & \\
\hline \multirow[t]{3}{*}{ Afecto positivo } & Entre grupos & 0,62 & 3 & 0,21 & \multirow[t]{3}{*}{1,85} & \multirow[t]{3}{*}{0,142} \\
\hline & Dentro de grupos & 13,06 & 116 & 0,11 & & \\
\hline & Total & 13,68 & 119 & & & \\
\hline \multirow[t]{3}{*}{ Afecto negativo } & Entre grupos & 0,81 & 3 & 0,27 & \multirow[t]{3}{*}{1,91} & \multirow[t]{3}{*}{0,132} \\
\hline & Dentro de grupos & 16,37 & 116 & 0,14 & & \\
\hline & Total & 17,18 & 119 & & & \\
\hline
\end{tabular}

Nota. Satisfacción vital Familia, Amigos y Sí mismo con Ajuste de Welch. 
Los resultados de las comparaciones planificadas para los dominios del bienestar subjetivo entre los grupos sin y con discapacidad se presentan en la Tabla 5.

Tabla 5

Comparaciones Planificadas de los Dominios del Bienestar Subjetivo entre los Grupos

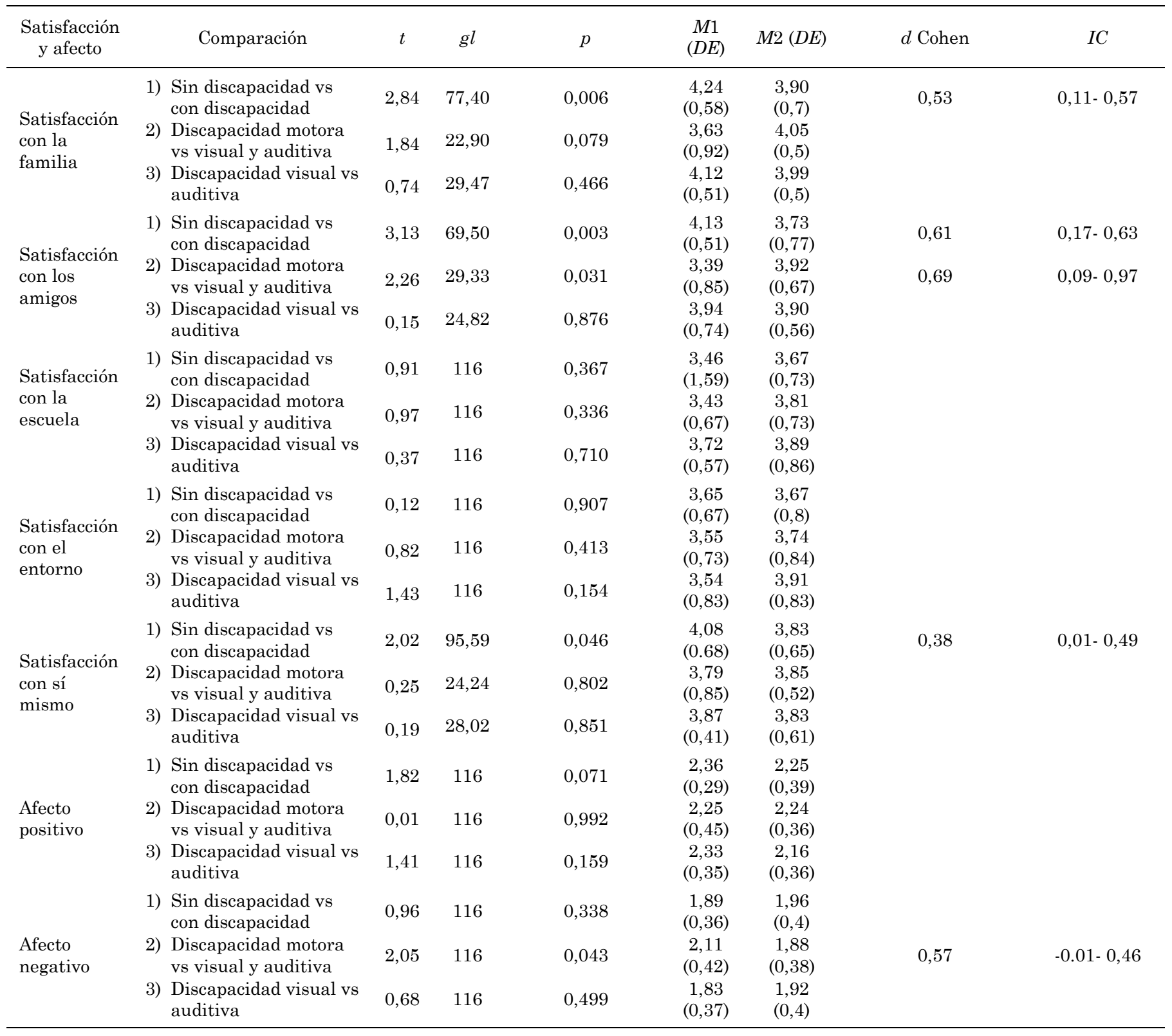

Nota. M1 y M2 corresponden a la media del primer y segundo grupo de la comparación, respectivamente.

En la Tabla 5 se observa que existen diferencias entre los estudiantes sin y con discapacidades en la satisfacción vital con la familia, siendo los estudiantes sin discapacidad los que se sienten más satisfechos que los con discapacidad en ese ámbito. Del mismo modo, se aprecian diferencias entre los grupos sin y con discapacidades en la satisfacción vital con los amigos, siendo los estudiantes sin discapacidades los que presentan mayor satisfacción que los grupos con discapacidades. Específicamente, en este último aspecto, se aprecian diferencias entre el grupo con discapacidad motora versus los grupos con discapacidad visual y auditiva, siendo los estudiantes con discapacidad motora los que se sienten menos satisfechos en este ámbito, en comparación a los otros dos grupos. 
Respecto a la satisfacción vital con el sí mismo, se observan diferencias entre los grupos sin y con discapacidades, evidenciando el primero una mayor satisfacción en este plano que los grupos con discapacidades. Finalmente, en cuanto al afecto negativo, se observan diferencias entre el grupo con discapacidad motora, en comparación a los grupos con discapacidad visual y auditiva, presentando el grupo con discapacidad motora una mayor afectividad negativa.

\section{Discusión}

Los hallazgos de esta investigación entregan evidencia que avala la relación entre la participación escolar y el bienestar subjetivo de escolares con y sin discapacidad. En primer término se observó una asociación directa y de tamaño moderado entre ambas variables; específicamente, destaca en todos los estudiantes (con y sin discapacidad) la asociación entre la participación escolar total y la percepción positiva que tiene el estudiante de las posibilidades de participación en su escuela, el afecto positivo global y la satisfacción que siente con su familia y consigo mismo.

En el caso de los estudiantes en situación de discapacidad, además, la participación se asocia con mayor satisfacción con la escuela. Este resultado es coherente con los resultados de estudios que indican que la participación escolar se constituye en factor protector para el bienestar de niños y adolescentes con y sin discapacidades (Condly, 2006). La misma relación fue detectada en otras investigaciones con estudiantes en situación de discapacidad, en las que la percepción de apoyo y la inclusión de los docentes y los compañeros en las clases regulares se asocian con niveles más altos de bienestar subjetivo en este grupo (Díaz Llanes, 2001; Opdenakker \& Van Damme, 2000).

Respecto a las diferencias por género, en este estudio se replicó el hallazgo de menores niveles de participación escolar de mujeres en situación de discapacidad, en comparación con la participación escolar de los hombres en esta situación. Estos resultados refuerzan lo reportado en el indicador participación escolar y formación ciudadana del Sistema Nacional de Evaluación de Resultados de Aprendizaje (SIMCE) 2016 para estudiantes en general, donde se señala que las mujeres disminuyen progresivamente el nivel de participación a medida que aumenta el grado escolar: $76 \%$ de alta participación en $4^{\circ}$ básico versus $51 \%$ de alta participación en $2^{\circ}$ medio ( 25 puntos de diferencia). En contraposición, en los hombres la diferencia porcentual en el nivel de alta participación escolar es de 14 puntos porcentuales (Chile, Ministerio de Educación, 2017).

En este estudio no se detectaron diferencias según NSE. Lo anterior puede deberse a que más del 50\% de la muestra pertenecía a NSE medio alto o alto. Al respecto, sería interesante continuar profundizando en este tema. El primer estudio nacional sobre la discapacidad (ENDISC) — que determinó el perfil de los chilenos en situación de discapacidad-, logró establecer la asociación entre situación de discapacidad, pobreza, exclusión educacional y menor participación en la sociedad y en la familia (Fondo Nacional de la Discapacidad \& Instituto Nacional de Estadísticas [FONADIS-INE], 2005). En otras palabras, las PeSD tienen menores oportunidades de participación que las personas que no tienen discapacidades (Cuevas \& Bunger, 2010).

Tampoco se detectaron diferencias en los distintos ámbitos de la participación escolar entre los grupos sin y con discapacidad. Este resultado difiere de lo reportado por otros autores (Eriksson et al., 2007; Eriksson \& Granlund, 2004), quienes indican que la participación de estudiantes con discapacidades está restringida, en comparación con sus pares sin discapacidad. Esto se evidencia en los menores niveles de participación en actividades estructuradas (actividades académicas) y no estructuradas (durante el recreo), existiendo también niveles de participación desigual entre los distintos tipos de discapacidades. El resultado de este estudio difiere de lo reportado en el segundo estudio nacional sobre la discapacidad [ENDISC II] (Chile, Ministerio de Desarrollo Social, 2016). En este informe se concluye que las PeSD en Chile participan en menor proporción que las personas sin discapacidad en actividades sociales tales como organizaciones sociales, centros de alumnos, voluntariados y organizaciones artísticas, entre otras, y que estos niveles disminuyen aún más en el caso de personas con discapacidades graves.

La ausencia de diferencias en los niveles de participación de los distintos grupos puede deberse a la baja potencia alcanzada en el estudio, debido al bajo tamaño muestral de los grupos con discapacidades, pero también a que el tamaño del efecto de la participación escolar en general, en esta muestra fue pequeño en comparación con otras investigaciones (Pérez-Salas et al., 2019). Así, los niveles de participación obtenidos en la muestra sin discapacidad son relativamente bajos en todas las dimensiones. La mayoría de ellos se 
ubicaron en la categoría de participación pocas veces (escala Likert opción $>2$ y $<3$ ) y solo la dimensión "percepción de oportunidades de participación escolar" se ubicó en la categoría algunas veces (escala Likert opción = 3). Esto contrasta con los resultados reportados en la muestra de validación, en la que los promedios en todas las dimensiones se ubicaron por sobre la categoría de participación algunas veces (escala Likert opción > 3 y <4) (Pérez-Salas et al., 2019).

Asimismo, el instrumento utilizado recoge la autopercepción de participación y de las oportunidades de participación en la escuela. Por tanto, la ausencia de diferencias entre los grupos puede ser explicada por el hecho de que todos los estudiantes considerados en el estudio eran parte de un PIE. En ese sentido, es posible que perciban que están participando en las distintas instancias de la vida escolar de una manera similar que estudiantes sin discapacidades (aunque sea baja). Esta percepción contrasta con los resultados de un estudio reciente, donde se señala que menos del $50 \%$ de los establecimientos reporta la incorporación de estudiantes en situación de discapacidad en los centros de alumnos, así como en la toma de decisiones relacionadas con el colegio (Tamayo Rozas et al., 2018).

Lo anterior tiene sentido si se analizan las diferencias encontradas entre la satisfacción vital reportada por los estudiantes con y sin discapacidades. Esto evidencia que la participación de manera equivalente a otros estudiantes no garantiza la satisfacción. Específicamente, en este estudio se observaron diferencias en la satisfacción en los ámbitos de la familia y amigos, siendo los estudiantes en situación de discapacidad los que menos satisfechos se sienten. La menor satisfacción vital con la familia puede deberse a la tendencia, reportada por algunos estudios (Ho et al., 2008; Sanders, 2006), que tendrían las familias de estudiantes con discapacidades de sobreprotegerlos y coartar la autonomía, lo que ha sido asociado a una menor satisfacción vital en estos grupos. Dicha hipótesis no fue explorada en este estudio, por lo que futuras investigaciones podrían evaluar esta posibilidad.

Asimismo, respecto al ámbito de los amigos, se observó que los estudiantes con trastorno motor son el grupo que menos satisfecho se siente con el grupo de pares. Lo anterior se condice con lo reportado por la revisión sistemática de Tough, Siegrist y Fekete (2017), donde se señala que el funcionamiento familiar, las interacciones sociales negativas y la calidad de las relaciones de amistad evidencian asociaciones consistentes con el bienestar de las personas con discapacidad física. En este sentido, los hallazgos de este estudio son un aporte al conocimiento en esta materia, puesto que, tal como reconocen Tough et al. (2017), existen pocos estudios disponibles en esta temática.

Los resultados anteriores también son concordantes con lo señalado por Moreira et al. (2015), respecto a que las PeSD perciben más negativamente los aspectos específicos de sus vidas, en comparación con las personas sin discapacidad. En esa misma línea, Brantley, Huebner y Nagle (2002) reportan que uno de los componentes del bienestar subjetivo (la satisfacción con la vida) es menor en niños en situación de discapacidad. De acuerdo con Gilman, Easterbrooks y Frey (2004), la relación entre la discapacidad y la satisfacción vital estaría mediada por el grado de inclusión social y educativa.

Entre las fortalezas que tiene este estudio se puede mencionar la entrega de información relevante sobre la participación y el bienestar subjetivo de estudiantes en situación de discapacidad en Chile. Estas variables son fundamentales porque impactan en la superación de las barreras presentadas en diversos contextos. Si bien existen estudios internacionales sobre la participación escolar y el bienestar subjetivo de escolares en situación de discapacidad, aún falta claridad en el conocimiento de diferencias existentes según los tipos de discapacidad (Moreira et al., 2015; OMS, 2011). Esto es más evidente en Chile, donde incluso los estudios realizados a nivel gubernamental sobre la discapacidad -ENDISC_ (FONADIS-INE, 2005) y ENDISC II (Chile, Ministerio de Desarrollo Social, 2016) presentan datos globales, sin desagregar por tipo.

La profundización en estos temas es relevante por dos motivos. El primero porque permitirá identificar la experiencia subjetiva de los estudiantes respecto de su participación en la escuela y su relación con el bienestar subjetivo. El segundo porque los resultados obtenidos permitirán a los establecimientos educacionales proponer intervenciones específicas que satisfagan las necesidades educativas especiales de sus estudiantes en situación de discapacidad y, por lo tanto, promover su funcionamiento positivo (Moreira et al., 2015). Asimismo, sería valioso realizar estudios longitudinales que permitieran evaluar el desarrollo de dichas variables a lo largo del periodo escolar.

Entre las principales limitaciones de este estudio, se encuentra el bajo tamaño muestral alcanzado en los grupos en situación de discapacidad evaluados. Por un lado, la muestra contemplada constituye un colectivo difícil de reclutar, puesto que la mayoría de los PIE del país se han enfocado principalmente en discapacidades de índole cognitiva y de aprendizaje (Tamayo Rozas et al., 2018). Por otro lado, los colegios, 
de acuerdo al Decreto 170 (Chile, Ministerio de Educación, 2009), reciben subvención por un máximo de dos estudiantes con necesidades educativas especiales permanentes por curso. Esto implica que debe contactarse a un número muy elevado de colegios para reclutar una muestra como la contemplada en la presente investigación.

Otra limitación fue la diferencia en el modo de aplicación de los instrumentos: mientras que los escolares sin discapacidad respondieron de manera autónoma, los escolares en situación de discapacidad fueron apoyados por una profesora diferencial. Esta profesional leyó las preguntas a las personas con discapacidad visual o motora y les tradujo el instrumento a LSCh a las personas con discapacidad auditiva que no podían leer fluidamente. Esta diferencia en el modo de aplicación y respuesta de los instrumentos podría ser una variable interviniente no controlada que pudo haber afectado los resultados. Sin embargo, tiene la ventaja de posibilitar la inclusión de grupos que, de lo contrario, quedarían marginados de la evaluación producto de la ausencia de instrumentos validados en lengua de señas y braille. Esto es central, pues según Parsons, Baum, Johnson y Hendershot (2001), se ha prestado escasa atención a cómo los procedimientos estándar de evaluación excluyen sistemáticamente a las PeSD de la investigación social realizada en población general. En esta investigación se implementó la estrategia de autorreporte asistido, el cual, de acuerdo a Kaye (2007, citado en Wilson et al., 2013), es útil cuando las PeSD tienen dificultades para responder cuestionarios. Por ello, el ofrecimiento de ayuda permite incrementar la tasa de respuesta en estas poblaciones. Asimismo, este autor argumenta que dicha estrategia debiera ser preferida a la exclusión de la investigación de los grupos con discapacidad o a la obtención de respuestas a través de un cuidador o proxy, puesto que permite recoger las respuestas desde su propia perspectiva.

Si bien se reconoce el valor que tiene esta investigación por incluir a PeSD, para comparar sus visiones con sus pares sin discapacidad, existe consciencia de que utilizar distintos métodos de evaluación (autorreporte, entrevista asistida y traducida a LSCh) tiene inconvenientes, siendo el principal la posible alteración de las propiedades psicométricas de los instrumentos utilizados. Sin embargo, hasta donde se tiene conocimiento, no existen instrumentos que permitan evaluar los constructos abordados en esta investigación con un método único para personas con discapacidad visual, auditiva y motora y sin discapacidades, de modo que la estrategia aquí utilizada era preferible a la no inclusión de estos grupos. Para comprobar la invarianza métrica de los distintos métodos utilizados sería necesaria una nueva investigación que probara tal hipótesis. Se expone aquí también el desafío de crear instrumentos de evaluación inclusivos que permitan la comparación de grupos con distintas discapacidades y no marginarlos también desde la investigación.

\section{Referencias}

Al Ju'beh K. (2017). Disability inclusive development toolkit. Bensheim, Alemania: CBM. Extraído de https://www.cbm.org/fileadmin/user_upload/Publications/CBM-DID-TOOLKIT-accessible.pdf

Antonak, R. F. \& Livneh, H. (2000). Measurement of attitudes towards persons with disabilities. Disability and Rehabilitation, 22, 211-224. https://doi.org/10.1080/096382800296782

Bax, M., Goldstein, M., Rosenbaum, P., Leviton, A., Paneth, N., Dan, B., Jacobsson, B. \& Damiano, D. (2005). Proposed definition and classification of cerebral palsy. Developmental Medicine \& Child Neurology, 47, 571-576. https://doi.org/10.1017/s001216220500112x

Brantley, A., Huebner, E. S. \& Nagle, R. J. (2002). Multidimensional life satisfaction reports of adolescents with mild mental disabilities. Mental Retardation, 40, 321-329. https://doi.org/10.1352/0047-6765(2002)040<0321:MLSROA>2.0.CO;2

Bunch, G. \& Valeo, A. (2004). Student attitudes toward peers with disabilities in inclusive and special education schools. Disability \& Society, 19, 61-76. https://doi.org/10.1080/0968759032000155640

Carona, C., Moreira, H., Silva, N., Crespo, C. \& Canavarro, M. C. (2014). Social support and adaptation outcomes in children and adolescents with cerebral palsy. Disability and Rehabilitation, 36, 584-592. https://doi.org/10.3109/09638288.2013.804596

Chaná, P. \& Alburquerque, D. (2006). La clasificación internacional del funcionamiento, de la discapacidad y de la salud (CIF) y la práctica neurológica. Revista Chilena de Neuro-Psiquiatría, 44, 89-97. https://doi.org/10.4067/S0717-92272006000200002

Chavarría, M. P. \& Barra, E. (2014). Satisfacción vital en adolescentes: relación con la autoeficacia y el apoyo social percibido. Terapia Psicológica, 32, 41-46. https://doi.org/10.4067/S0718-48082014000100004

ChildFund International (2016). Gender-based violence against children and youth with disabilities: A toolkit for child protection actors. Richmond, VA: Autor. Extraído de https://resourcecentre.savethechildren.net/node/13841/pdf/gbv-against-children-andyouth-with-disabilities-toolkit.pdf

Chile, Ministerio de Desarrollo Social (2016). Segundo estudio nacional de la discapacidad 2015. Santiago, Chile: Autor, Servicio Nacional de la $\quad$ Discapacidad. http://observatorio.ministeriodesarrollosocial.gob.cl/endisc/docs/Libro_Resultados_II_Estudio_Nacional_de_la_Discapacidad.pdf

Chile, Ministerio de Educación (2009). Decreto $N^{o}$ 170/2009: fija normas para determinar los alumnos con necesidades educativas especiales que serán beneficiarios de las subvenciones para educación especial. Santiago, Chile: Autor. Extraído de https://especial.mineduc.cl/wp-content/uploads/sites/31/2018/06/DTO-170_21-ABR-2010.pdf

Chile, Ministerio de Educación (2017). Estadísticas de la educación 2016. Santiago, Chile: Autor, División de Planificación y Presupuesto, Centro de Estudios. Extraído de https://centroestudios.mineduc.cl/wp-content/uploads/sites/100/2017/07/Anuario_2016.pdf 
Condly, S. J. (2006). Resilience in children: A review of literature with implications for education. Urban Education, 41, 211-236. https://doi.org/10.1177/0042085906287902

Cuevas, G. \& Bunger, S. (2010). Epidemiología de la discapacidad y desarrollo de la red de rehabilitación en la última década. Revista Hospital Clínico Universidad de Chile, 21, 289-297. Extraído de https://www.redclinica.cl/Portals/0/Users/014/14/14/Publicaciones/epidemiol_discapacidad.pdf

Díaz Llanes, G. (2001). El bienestar subjetivo. Actualidad y perspectivas. Revista Cubana de Medicina General Integral, $17,572-579$. Extraído de http://scielo.sld.cu/scielo.php?script=sci_arttext\&pid=S0864-21252001000600011\&lng=es\&nrm=iso\&tlng=es

Diener, E., Wirtz, D., Biswas-Diener, R., Tov, W., Kim-Prieto, C., Choi, D. -W. \& Oishi, S. (2009). New measures of well-being. En E. Diener (Ed.), Assessing well-being: The collected works of Ed Diener (pp. 247-266). Dordrecht, Países Bajos: Springer.

Domínguez, A. B. (2009). Educación para la inclusión de alumnos sordos. Revista Latinoamericana de Educación Inclusiva, 3(1), 45-51. Extraído de https://sid.usal.es/idocs/F8/ART11921/educacion_para_la_inclusion_de_alum_sordos.pdf

Egilson, S. T. \& Coster, W. J. (2004) School function assessment: Performance of Icelandic students with special needs. Scandinavian Journal of Occupational Therapy, 11, 163-170. https://doi.org/10.1080/11038120410020737

Eriksson, L. \& Granlund, M. (2004). Conceptions of participation in students with disabilities and persons in their close environment. Journal of Developmental and Physical Disabilities, 16, 229-245. https://doi.org/10.1023/B:JODD.0000032299.31588.fd

Eriksson, L., Welander, J. \& Granlund, M. (2007). Participation in everyday school activities for children with and without disabilities. Journal of Developmental and Physical Disabilities, 19, 485-502. https://doi.org/10.1007/s10882-007-9065-5

Fernández-López, J. A., Fernández-Fidalgo, M., Geoffrey, R., Stucki, G. \& Cieza, A. (2009). Funcionamiento y discapacidad: la clasificación internacional del funcionamiento (CIF). Revista Española de Salud Pública, 83, 775-783. https://doi.org/10.1590/s1135-57272009000600002

Field, A. (2009). Discovering statistics using SPSS ( $3^{\mathrm{a}}$ ed.). London, United Kingdom: Sage.

Fondo Nacional de la Discapacidad \& Instituto Nacional de Estadísticas (2005). Primer estudio nacional de la discapacidad en Chile. Santiago, Chile: Fondo Nacional de la Discapacidad. Extraído de https://www.senadis.gob.cl/descarga/i/3011

Gilman, R., Easterbrooks, S. R. \& Frey, M. (2004). A preliminary study of multidimensional life satisfaction among deaf/hard of hearing youth across environmental settings. Social Indicators Research, 66, 143-164. https://doi.org/10.1023/B:SOCI.0000007495.40790.85

Godoy, M. P., Meza, M. L. \& Salazar, A. (2004). Antecedentes históricos, presente y futuro de la educación especial en Chile. Santiago, Chile: Ministerio de Educación. Extraído de http://especial.mineduc.cl/wp-content/uploads/sites/31/2016/08/201304151210180.doc_Antecedentes_Ed_Especial.pdf

Gómez-Vela, M. \& Verdugo, M. A. (2004). El cuestionario de evaluación de la calidad de vida de alumnos de educación secundaria obligatoria: descripción, validación inicial y resultados obtenidos tras su aplicación en una muestra de adolescentes con discapacidad y sin ella. Siglo Cero: Revista Española sobre Discapacidad Intelectual, 35(4), 5-17. Extraído de https://sid.usal.es/idocs/F8/ART6809/articulos1.pdf

González, M., Oyanadel, C. \& Peñate, W. (2018). Adaptación y validación de la Escala de Afecto Positivo y Negativo en niños y adolescentes chilenos. Manuscrito sometido para publicación.

Hair, J. F., Anderson, R. E., Tatham, R. L. \& Black, W. C. (2005). Análisis multivariante (5a ed.). Madrid: Prentice Hall.

Hein, S., Grumm, M. \& Fingerle, M. (2011). Is contact with people with disabilities a guarantee for positive implicit and explicit attitudes? European Journal of Special Needs Education, 26, 509-522. https://doi.org/10.1080/08856257.2011.597192

Hemmingsson, H. \& Borell, L. (2000). Accommodation needs and student-environment fit in upper secondary schools for students with severe physical disabilities. Canadian Journal of Occupational Therapy, 67, 162-172. https://doi.org/10.1177/000841740006700311

Hemmingsson, H. \& Borell, L. (2002). Environmental barriers in mainstream schools. Child: Care, Health, and Development, 28, 5763. https://doi.org/10.1046/j.1365-2214.2002.00240.x

Hemmingsson, H., Gustavsson, A. \& Townsend, E. (2007). Students with disabilities participating in mainstream schools: Policies that promote and limit teacher and therapist cooperation. Disability \& Society, 22, 383-398. https://doi.org/10.1080/09687590701337892

Hernández Sampieri, R., Fernández-Collado, C. \& Baptista Lucio, M. P. (2006). Metodología de la investigación (4ª ed.). México DF, México: McGraw-Hill.

Ho, S. M. Y., Fung, B. K. K., Fung, A. S. M., Chow, S. P., Ip, W. Y., Lee, S. F. Y. ... Ha, K. W. Y. (2008). Overprotection and the psychological states of cerebral palsy patients and their caretakers in Hong Kong: A preliminary report. Hong Kong Medical Journal, 14, 286-291. Extraído de https://www.researchgate.net/profile/Boris_Fung/publication/23154104_Overprotection_and_the_psychological_states_of_cerebral_palsy_pati ents_and_their_caretakers_in_Hong_Kong_A_preliminary_report/links/Ofcfd50c6ca5b4a6eb000000/Overprotection-and-the-psychologicalstates-of-cerebral-palsy-patients-and-their-caretakers-in-Hong-Kong-A-preliminary-report.pdf

Huebner, E. S. (2001). Manual for the Multidimensional Students' Life Satisfaction Scale. Columbia: University of South Carolina.

Ibáñez, A., Becerra, C., López, V., Sirlopú, D. \& Cornejo, C. (2005). Iconicidad y metáfora en el lenguaje chileno de signos (LENSE): un análisis cualitativo. Relieve: Revista Electrónica de Investigación y Evaluación Educativa, 11, 27-45. https://doi.org/10.7203/relieve.11.1.4195

IBM (2017). IBM SPSS Statistics for Windows, version 25.0. Armonk, NY: IBM Corp.

John-Akinola, Y. O. \& Nic-Gabhainn, S. (2014). Children's participation in school: A cross-sectional study of the relationship between school environments, participation and health and well-being outcomes. BMC Public Health, 14,964 . https://doi.org/10.1186/1471-2458-14-964

Kef, S., Hox, J. J. \& Habekothé, H. T. (2000). Social networks of visually impaired and blind adolescents. Structure and effect on wellbeing. Social Networks: An International Journal of Structural Analysis, 22, 73-91. https://doi.org/10.1016/S0378-8733(00)00022-8

King, G., Lawm, M., King, S., Rosenbaum, P., Kertoy, M. K. \& Young, N. L. (2003). A conceptual model of the factors affecting the recreation and leisure participation of children with disabilities. Physical \& Occupational Therapy in Pediatrics, 23(1), 63-90. https://doi.org/10.1080/J006v23n01_05

Konu, A. I., Lintonen, T. P. \& Rimpelä, M. K. (2002). Factors associated with schoolchildren's general subjective well-being. Health Education Research, 17, 155-165. https://doi.org/10.1093/her/17.2.155

Law, M. (2002). Participation in the occupations of everyday life. American Journal of Occupational Therapy, 56, 640-649. https://doi.org/10.5014/ajot.56.6.640

Mancini, M. C., Coster, W. J., Trombly, C. A. \& Heeren, T. C. (2000). Predicting elementary school participation in children with disabilities. Archives of Physical Medicine and Rehabilitation, 81, 339-347. https://doi.org/10.1016/S0003-9993(00)90081-9

Manghi, D., Julio, C., Conejeros, M. L., Donoso, E., Murillo, M. L. \& Diaz, C. (2012). El profesor de educación diferencial en Chile para el siglo XXI: tránsito de paradigma en la formación profesional. Perspectiva Educacional, 51(2), 46-71. https://doi.org/10.4151/07189729-Vol.51-Iss.2-Art.109 
Matos, M. G. de \& Carvalhosa, S. F. (2001). A saúde dos adolescentes: ambiente escolar e bem-estar [Salud del adolescente: ambiente escolar y bienestar]. Psicologia, Saúde \& Doenças, 2, 43-53. Recuperado de http://www.scielo.mec.pt/scielo.php?script=sci_abstract\&pid=S1645$00862001000200003 \& \operatorname{lng}=$ pt\&nrm=iso

Mehnert, T., Krauss, H. H., Nadler, R. \& Boyd, M. (1990). Correlates of life satisfaction in those with disabling conditions. Rehabilitation Psychology, 35, 3-17. https://doi.org/10.1037/h0079046

Michailakis, D. (2003). The systems theory concept of disability: One is not born a disabled person, one is observed to be one. Disability \& Society, 18, 209-229. https://doi.org/10.1080/0968759032000044184

Moreira, P. A. S., Bilimoria, H., Alvez, P., Santos, M. A., Macedo, A. C., Maia. A. ... Miranda, M. J. (2015). Subjective wellbeing in students with special educational needs. Cognition, Brain, Behavior, 19, 75-97. $97 . \quad$ Extraído de https://www.tandfonline.com/doi/abs/10.1080/1034912X.2016.1144873

Morris, C., Kurinczuk, J. J., Fitzpatrick, R. \& Rosenbaum, P. L. (2006). Do the abilities of children with cerebral palsy explain their activities and participation? Developmental Medicine \& Child Neurology, 48, 954-961. https://doi.org/10.1111/j.1469-8749.2006.tb01265.x

Muñoz Villa, M. L., López Cruz, M. \& Assaél, J. (2015). Concepciones docentes para responder a la diversidad: ¿barreras o recursos para la inclusión educativa? Psicoperspectivas, 14(3), 68-79. https://doi.org/10.5027/psicoperspectivas-Vol14-Issue3-fulltext-646

Nunes, T., Pretzlik, U. \& Olsson, J. (2001). Deaf children's social relationships in mainstream schools. Deafness \& Education International, 3, 123-136. https://doi.org/10.1179/146431501790560972

Opdenakker, M. -C. \& Van Damme, J. (2000). Effects of school, teaching staff and classes on achievement and well-being in secondary education: Similarities and differences between school outcomes. School Effectiveness and School Improvement, 11, 165-196. https://doi.org/10.1076/0924-3453(200006)11:2;1-Q;FT165

Organización de las Naciones Unidas (2006). Convención sobre los derechos de las personas con discapacidad y protocolo facultativo. New York, NY: Autor. Extraído de http://www.un.org/disabilities/documents/convention/convoptprot-s.pdf

Organización Mundial de la Salud (2001). Clasificación internacional del funcionamiento de la discapacidad y de la salud: CIF. Madrid, España: Ministerio de Trabajo y Asuntos Sociales, Secretaría General de Asuntos Sociales, Instituto de Migraciones y Servicios Sociales. Extraído de http://www.imserso.es/InterPresent2/groups/imserso/documents/binario/435cif.pdf

Organización Mundial de la Salud (2011). Resumen: informe mundial sobre la discapacidad. Il-Belt Valletta, Malta: Autor. Extraído de https://www.who.int/disabilities/world_report/2011/summary_es.pdf

Palacios, A. (2008). El modelo social de discapacidad: orígenes, caracterización y plasmación en la Convención Internacional sobre los Derechos de las Personas con Discapacidad (Colección CERMI N 36). Madrid, España: Comité Español de Representantes de Personas con Discapacidad. Extraído de https://www.cermi.es/sites/default/files/docs/colecciones/Elmodelosocialdediscapacidad.pdf

Paolinelli, G. C. \& González, A. M. (2014). Epidemiología de la discapacidad en Chile, niños y adultos. Revista Médica Clínica Las Condes, 25, 177-182. https://doi.org/10.1016/S0716-8640(14)70028-8

Parsons, J. A., Baum, S., Johnson, T. P. \& Hendershot, G. (2001). Inclusion of disabled populations in interview surveys: Review and recommendations. En S. N. Barnartt \& B. M. Altman (Eds.), Exploring Theories and Expanding Methodologies: Where we are and where we need to go (Research in Social Science and Disability, Vol. 2), (pp. 167-184). Bingley, United Kingdom: Emerald Group Publishing. https://doi.org/10.1016/S1479-3547(01)80025-7.

Pérez-Salas, C. P., Sirlopú, D., Cobo, R. \& Awad, A. (2019). Análisis bifactorial de la Escala de Participación Escolar en una muestra de estudiantes chilenos. Revista Iberoamericana de Diagnóstico y Evaluación/e Avaliaçao Psicológica, 52(3), 27-39. https://doi.org/10.21865/RIDEP52.3.03

Pianta, R. C. (2006). Classroom management and relationships between children and teachers: Implications for research and practice. En C. M. Evertson \& C. S. Weinstein (Eds.), Handbook of classroom management: Research, practice, and contemporary issues (pp. 685-709). Mahwah, NJ: Lawrence Erlbaum.

Polo Sánchez, M. T., Fernández Jiménez, C. \& Díaz Batanero, C. (2011). Estudio de las actitudes de estudiantes de ciencias sociales y psicología: relevancia de la información y contacto con las personas discapacitadas. Universitas Psychologica, 10(1), 113-124. https://doi.org/10.11144/Javeriana.upsy10-1.eaec

Ramos, L. (2013). Educación especial y educación inclusiva en Chile: ¿en punto de estancamiento? Revista Latinoamericana de Educación Inclusiva, 7(2), 37-46. Extraído de http://www.rinace.net/rlei/numeros/vol7-num2/art2.pdf

Rodríguez Martín, A. \& Álvarez Arregui, E. (2013). Development and validation of a scale to identify attitudes towards disability in higher education. Psicothema, 25, 370-376. https://doi.org/10.7334/psicothema2013.41

Sánchez Bravo, A., Díaz Flores, C., Sanhueza Henríquez, S. \& Friz Carrillo, M. (2008). Percepciones y actitudes de los estudiantes de pedagogía hacia la inclusión educativa. Estudios Pedagógicos, 34(2), 169-178. https://doi.org/10.4067/S0718-07052008000200010

Sanders, K. Y. (2006). Overprotection and lowered expectations of persons with disabilities: The unforeseen consequences. Work, 27, 181-188. Extraído de https://content.iospress.com/articles/work/wor00561

Sandín, B. (2003). Escalas PANAS de Afecto Positivo y Negativo para Niños y Adolescentes (PANASN). Revista de Psicopatología y Psicología Clínica, 8, 173-182. https://doi.org/10.5944/rppc.vol.8.num.2.2003.3953

Sandín, B., Chorot, P., Lostao, L., Joiner, T. E., Santed, M. A. \& Valiente, R. M. (1999). Escalas PANAS de Afecto Positivo y Negativo: validación factorial y convergencia transcultural. Psicothema, 11, 37-51. Extraído de http://www.psicothema.com/psicothema.asp?id=229

Simeonsson, R. J., Carlson, D., Huntington, G. S., McMillen, J. S. \& Brent, J. L. (2001). Students with disabilities: A national survey of participation in school activities. Disability and Rehabilitation, 23, 49-63. https://doi.org/10.1080/096382801750058134

Smith, B. \& Bundon, A. (2018). Disability models: Explaining and understanding disability sport in different ways. En I. Brittain \& A. Beacom (Eds.), The Palgrave handbook of paralympic studies (pp. 15-34). London, Reino Unido: Palgrave Macmillan.

Steptoe, A., Deaton, A. \& Stone, A. A. (2015). Subjective wellbeing, health, and ageing. The Lancet, 385, 640648. https://doi.org/10.1016/S0140-6736(13)61489-0

Suriá Martínez, R. (2016). Bienestar subjetivo, resiliencia y discapacidad. Acciones e Investigaciones Sociales, 36, 113-140. https://doi.org/10.26754/2016361490

Tamayo Rozas, M., Carvallo Arrau, M. F., Sánchez Cornejo, M., Besoaín-Saldaña. Á. \& Rebolledo Sanhueza, J. (2018). Programa de integración escolar en Chile: brechas y desafíos para la implementación de un programa de educación inclusiva. Revista Española de Discapacidad, 6(1), 161-179. https://doi.org/10.5569/2340-5104.06.01.08

Tenorio, S. (2011). Formación inicial docente y necesidades educativas especiales. Estudios Pedagógicos, 37(2), 249-265. https://doi.org/10.4067/S0718-07052011000200015 
Tinklin, T., Croxford, L., Ducklin, A. \& Frame, B. (2003). Inclusion: A gender perspective. Policy Futures in Education, 1, 640-652. https://doi.org/10.2304/pfie.2003.1.4.3

Thomas, C. (2007). Sociologies of disability and illness. Contested ideas in disability studies and medical sociology. London, Reino Unido: Red Globe Press.

Torres Gutiérrez, A. L. (2006). Atención al educando ciego o con deficiencias visuales. San José, Costa Rica: Editorial Universidad Estatal a Distancia.

Tough, H., Siegrist, J. \& Fekete, C. (2017). Social relationships, mental health and wellbeing in physical disability: A systematic review. BMC Public Health, 17, article 414. https://doi.org/10.1186/s12889-017-4308-6

Watson, D., Clark, L. A. \& Tellegen, A. (1988). Development and validation of brief measures of positive and negative affect: The PANAS Scales. Journal of Personality and Social Psychology, 54, 1063-1070. https://doi.org/10.1037/0022-3514.54.6.1063

Wilson, E., Campain, R., Moore, M., Hagiliassis, N., McGillivray, J., Gottliebson, D. ... \& Graffam, J. (2013). An accessible survey method: Increasing the participation of people with a disability in large sample social research. Telecommunications Journal of Australia, 63(2), 24.1-24.13. https://doi.org/10.7790/tja.v63i2.411

Fecha de recepción: Julio de 2019.

Fecha de aceptación: Septiembre de 2019. 